\title{
CARESSES: The World's First Culturally Sensitive Robots for Elderly Care
}

\author{
Compiled by: Giuseppe Riva, $\mathrm{PhD}^{1,2}$ and Eleonora Riva, $\mathrm{PhD}^{3}$
}

This column will try to describe the characteristics of current cyberpsychology research in Europe. In particular, CyberEurope aims at describing the leading research groups and projects running on the other side of the Ocean.

$\mathbf{R}$ ESEARCHERS HAVE DEVELOPED revolutionary new robots that adapt to the culture and customs of the elderly people they assist.

Population ageing has implications for many sectors of society, one of which is the increased demand on a country's health and social care resources. This burden could be greatly eased through advances in artificial intelligence. Robots have the potential to provide valuable assistance to caregivers in hospitals and care homes. They could also improve home care and help the elderly live more independently. But to do this, they will have to be able to respond to older people's needs in a way that is more likely to be trusted and accepted.

The EU-funded project Culture Aware Robots and Environmental Sensor Systems for Elderly Support (CARESSES; http://caressesrobot.org/en/) has set out to build the first ever culturally competent robots to care for the elderly. The groundbreaking idea involved designing these robots to adapt their way of acting and speaking to match the culture and habits of the elderly person they're assisting.

"The idea is that robots should be capable of adapting to human culture in a broad sense defined by a person's belonging to a particular ethnic group. At the same time robots must be able to adapt to an individual's personal preferences so in that sense it doesn't matter if you're Italian or Indian,' explained researcher Alessandro Saffiotti of project partner Örebro University Sweden in a news item posted on the Phys.org Web site.

\section{A Robot that Adapts Itself to the User's Cultural Identity and Individual Characteristics}

Through its communication with an elderly person, the robot will fine-tune its knowledge by adapting to that person's cultural identity and individual characteristics. Using this knowledge, it will be able to remind the elderly person to take their prescribed medication, encourage them to eat healthily and be active, or help them stay in touch with family and friends. The robot will also be able to make suggestions about the appropriate clothing for specific occasions and remind people of upcoming religious and other celebrations. It doesn't replace a home care worker. Nevertheless, it will play a vital role in helping to make elderly people's lives less lonely and reducing the need to have a caregiver nearby at all times.

Scientists are testing the first CARESSES robots in care homes in the United Kingdom and Japan. They're being used to assist elderly people from different cultural backgrounds. The aim is to see if people feel more comfortable with robots that interact with them in a culturally sensitive manner. They're also examining whether such robots improve the elderly's quality of life. "The testing of robots outside of the laboratory environment and in interaction with the elderly will without a doubt be the most interesting part of our project," added Saffiotti.

The innovative CARESSES robots may pave the way for more culturally sensitive services beyond the sphere of elderly care too. "It will add value to robots intended to interact with people. Which is not to say that today's robots are completely culture-neutral. Instead, they unintentionally reflect the culture of the humans who build and program them."

Address correspondence to:

Dr. Giuseppe Riva

Department of Psychology

Catholic University of Milan

Largo Gemelli 1-20123 Milan

Italy

E-mail: giuseppe.riva@unicatt.it

Dr. Eleonora Riva Department of Cultural Heritage and Environment University of Milan Milan Italy

E-mail: eleonora.riva@unimi.it

Sources: Cordis, European Commission and European Union

\footnotetext{
${ }^{1}$ Department of Psychology, Catholic University of Milan, Milan, Italy.

${ }^{2}$ Applied Technology for Neuro-Psychology Lab, Istituto Auxologico Italiano, Milan, Italy.

${ }^{3}$ Department of Cultural Heritage and Environment, University of Milan, Milan, Italy.
} 